\title{
Segmented Infrared Image Analysis for Rotating Machinery Fault Diagnosis
}

\author{
Lixiang Duan, Mingchao Yao, Jinjiang Wang*, Tangbo Bai, Laibin Zhang
}

\begin{abstract}
As a noncontact and non-intrusive technique, infrared image analysis becomes promising for machinery defect diagnosis. However, the insignificant information and strong noise in infrared image limit its performance. To address this issue, this paper presents an image segmentation approach to enhance the feature extraction in infrared image analysis. A region selection criterion named dispersion degree is also formulated to discriminate fault representative regions from unrelated background information. Feature extraction and fusion methods are then applied to obtain features from selected regions for further diagnosis. Experimental studies on a rotor fault simulator demonstrate that the presented segmented feature enhancement approach outperforms the one from the original image using both Naïve Bayes classifier and support vector machine.
\end{abstract}

\section{Keywords:}

Infrared image analysis, feature enhancement, region selection, machinery fault diagnosis

Page 1 of 23

(C) 2016. This manuscript version is made available under the Elsevier user license http://www.elsevier.com/open-access/userlicense/1.0/ 


\section{INTRODUCTION}

Advances in modern manufacturing have led to better product quality, and increased flexibility and productivity [1]. Inevitably, machinery usually operates under high speed and heavy load conditions. Complex and severe operating conditions have posed significant challenges on machinery availability and reliability. Thus, machinery fault diagnosis is of great importance to ensure trouble-free operations [2]. Increasing demand on machinery reliability accelerates the integration of sensing techniques for machinery condition monitoring, such as vibration, oil analysis, and infrared image analysis, etc., of which the sensitivities to fault diagnosis are summarized in Fig. 1.

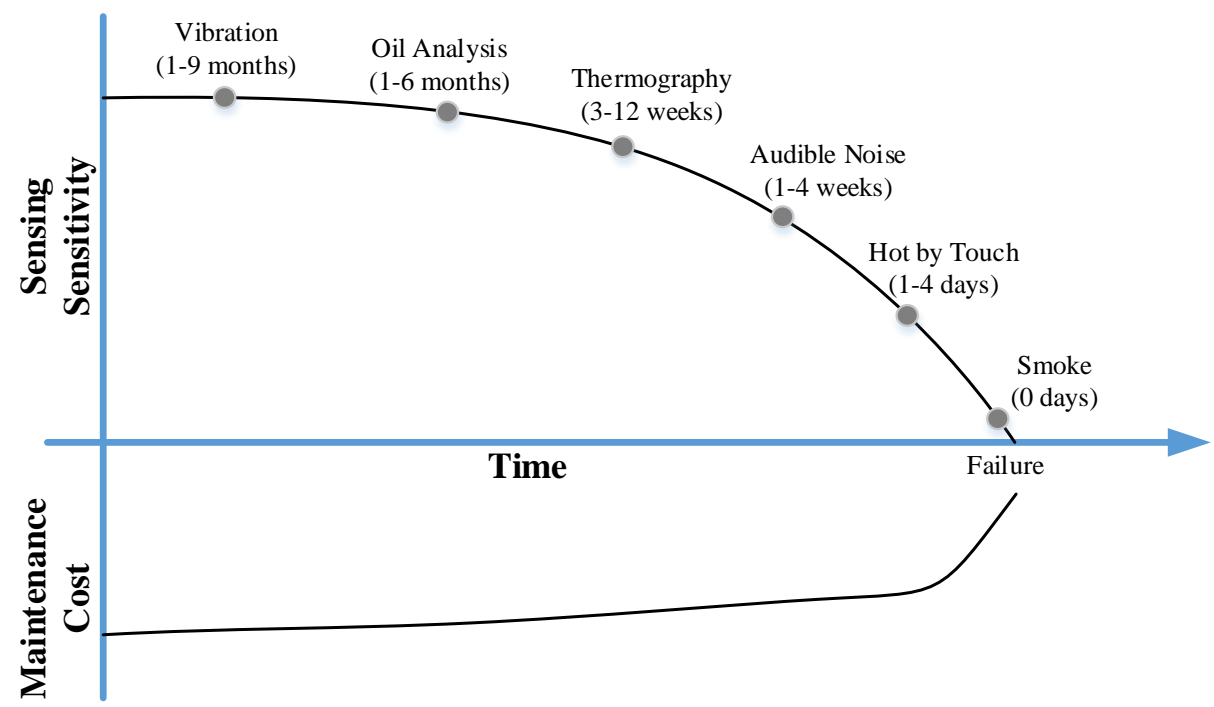

Fig. 1. Sensitivity comparison among different sensing techniques for machinery defect diagnosis and the corresponding maintenance cost (data from http://www.plantservices.com/).

Vibration analysis has been widely investigated, and a variety of signal processing techniques including wavelet transform [3], empirical mode decomposition [4], and Hilbert transform [5] have also been developed for machinery incipient defect diagnosis. However, 
vibration signal measurement is usually affected by the selection of sensor locations [6], harsh operating environments such as high temperature, and greasy surface which poses challenges on the installation of contact-type vibration sensors. Oil analysis could detect debris in the lubrication system for defect diagnosis, but it is usually performed offline [7].

As a non-contact and non-destructive technique, Infrared thermography (IRT) is a measurement methodology, where the infrared radiation (wavelength lies between 0.75 and 1000 lm) emitted from the surface of the object under inspection is recorded using an infrared camera. The temperature of the monitored object is calculated from the intensity of the emitted radiation using Stefan-Boltzmann's law [1-2]. Infrared thermal image analysis has attracted much interest for condition monitoring [8] by detecting temperature anomaly caused by heat, friction, and excessive vibration, etc. It has been exploited in a wide range of application scenarios of thermal image analysis, including machinery defect diagnosis [9-11], building monitoring [12], material/structure defect assessment [13, 14], medical inspection [15-17], and nondestructive testing [18-20]. Thermal image analysis usually involves feature extraction, feature selection/reduction, and classification. In [9] an integrative approach is investigated for rotating machinery defect diagnosis, including a two-dimensional discrete wavelet transform for feature extraction, the Mahalanobis distance and relief algorithm for feature selection, support vector machine and linear discriminant analysis for classification. In [21], genetic algorithm is investigated to select features extracted using 2D-DWT, and artificial neural network is then studied for cooling radiator fault diagnosis. Support vector machine is also investigated in [22] using the extracted histogram features from a thermal image for machinery defect diagnosis, and the classification accuracy of the thermal image is comparable to that of vibration signals. In [23], a bi-dimensional empirical mode decomposition (BEMD) is firstly used for image enhancement, 
and then generalized discriminant analysis (GDA) and relevance vector machine (RVM) are studied for feature reduction and fault classification, respectively.

Prior studies of infrared thermal image analysis usually take the whole image or cropped image as input for machinery defect diagnosis. However, most regions of the thermal image are unrelated background information; while the information of interest with temperature anomaly is often located in a small region corresponding to some specific parts, such as faulty bearing, bent shaft, and motors, etc. Such characteristic of insignificant information in infrared thermal image affects the efficiency of feature extraction and the accuracy of subsequent classification.

To enhance fault-related information and improve the accuracy of defect diagnosis, this paper presents a segmented infrared thermal image analysis method comparing with prior studies using original image. The image is segmented into a range of rectangular regions. A dispersion degree criterion is formulated to select fault related regions as the information of interest. Features are then extracted and fused for subsequent fault classification. Experimental studies on a rotor test rig demonstrate the effectiveness of the presented method by yielding higher classification accuracy using Naive Bayes classifier and support vector machine.

The contribution of this paper rest on two folds: 1) a segmented thermal image analysis method is presented, and experimental studies show the presented method yields higher classification accuracy comparing with prior studies using original image; and 2) a dispersion degree criterion is formulated for region selection, and its rationality is discussed from physical interpretation of machinery failure modes. The rest of the paper is organized as follows. The theoretical framework of the feature enhancement system is then presented in Section 2. Next, the effectiveness of the developed method is demonstrated in the experimental studies on a rotor test rig in Section 3. Finally, the conclusions are drawn in Section 4. To be self-contained, the 
theoretical background of Naïve Bayesian Classifier and Support Vector Machine are discussed and included in the Appendix section.

\section{THE PROPOSED METHOD}

The essential to fault diagnosis is to extract representative features insensitive to the background noise and improve the pattern classification accuracy. To enhance machine diagnosis accuracy of infrared image analysis, this paper presents a segmented infrared image analysis method for machinery defect diagnosis, and proposed a dispersion degree based region selection criterion to choose fault-related regions as the information of interest for subsequent defect classification. The segmented infrared image analysis method is illustrated in Fig. 2. It consists of several modules including data acquisition, image segment, region selection, feature extraction, feature fusion, and fault classification.

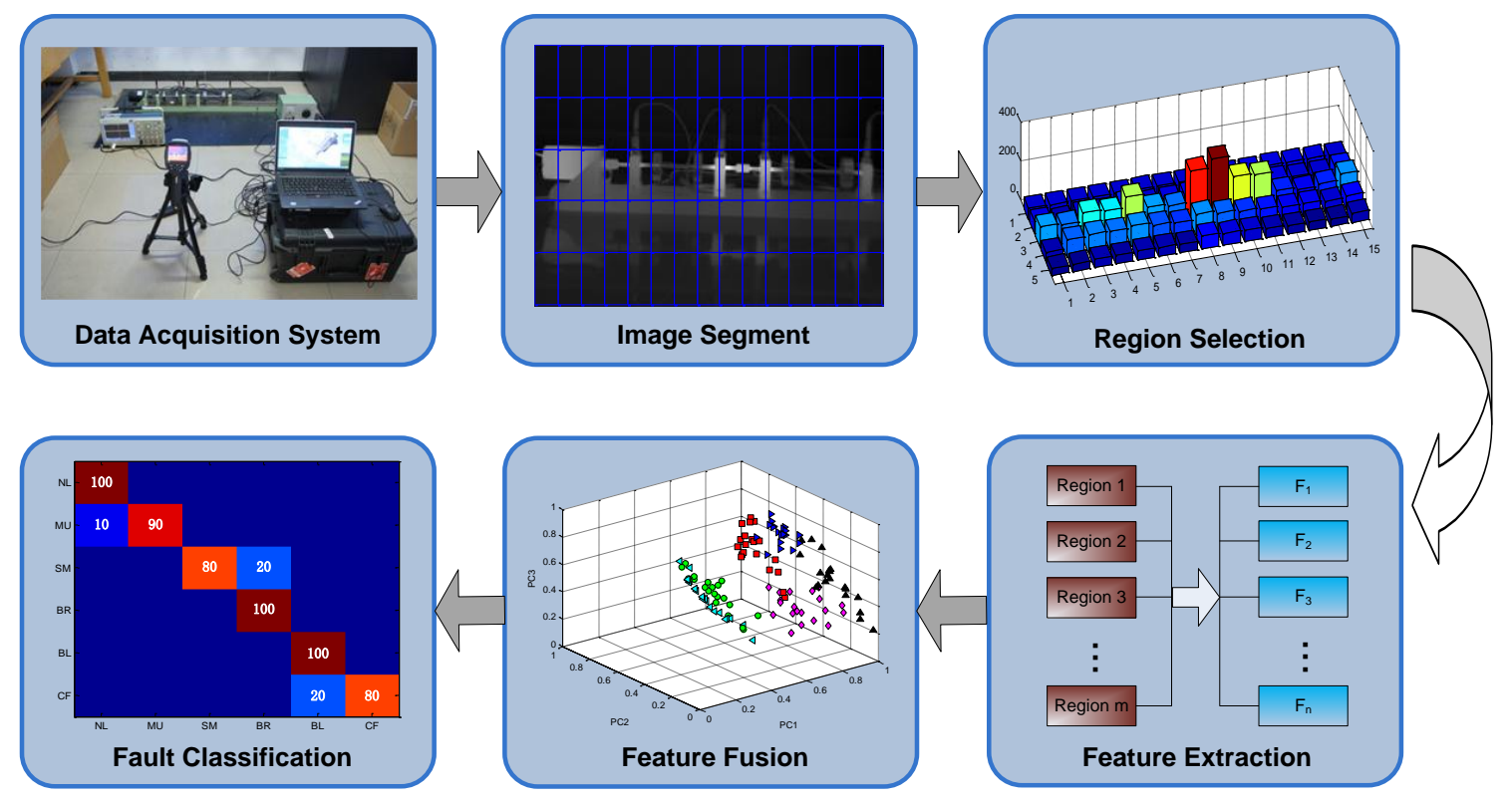

Fig. 2. The segmented infrared image analysis diagram for machinery defect diagnosis. 
The infrared images captured from the thermal camera are firstly segmented into rectangular regions of different divisions. Regions which are sensitive to faults are then picked out by dispersion degree criterion; one specific region is for a relevant independent fault. Then the feature extraction and the PCA feature fusion are carried out to extract the features and reduce the high dimension of the feature space, respectively. The selected features are then fed into classifiers (e.g. support vector machine and Naïve Bayes classifier) for machinery defect diagnosis. The segmented infrared image analysis method selects the fault-related regions to enhance feature contrast, and is expected to improve the classification accuracy.

\subsection{Image Segmentation and Region Selection}

To eliminate irrelevant background noise, an image is divided into different regions for fault-related region selection. For the sake of easy implementation, the original rectangular infrared image is first separated into a range of rectangular regions. It is obvious that region selection has great effect on the accuracy of fault diagnosis. According to the property of heat dissipation, the highest temperature usually occurs at the center of a specific region (corresponding to faulty component) and spread around the center point. Thus a differential temperature matrix is firstly obtained as the difference between infrared thermal image of fault condition and that of normal condition. The energy variance of each segment is calculated and derived from standard deviation. The value 0 indicates that there is no energy variance between faulty condition and normal condition in the specific region. Thus, dispersion degree is proposed to extract the representative region containing primary information. The dispersion degree 
criterion is formulated to describe the distribution of temperature difference as:

$$
d(r, c)=\sqrt{\frac{1}{r d \times c d-1} \sum_{r d} \sum_{c d}\left(T(r d, c d)-\frac{\sum_{r d} \sum_{c d} T(r d, c d)}{r d \times c d}\right)^{2}}
$$

where $d$ is the dispersion degree value. $d(r, c)$ represents the value at row $r$ and column $c . r d, c d$ is the resolution ratio of temperature difference matrix divided by row $r$ and column $c$. $T$ is the

matrix of temperature difference. $T(r d, c d)$ represents the temperature difference value at row $r d$ and column $c d$. By calculating the dispersion degree of each region, the fault related region with highest value is selected as the information of interest.

\subsection{Features Extraction and Selection}

Due to the high dimensional space of image, feature extraction and selection areusually performed for dimension reduction in infrared thermal image analysis. For digital image processing, grey histogram is commonly used to represent the relationship between image grey level and its statistical probability [24]; and histogram features can give the comprehensive descriptions of the image. The first-order histogram probability $P(g)$ of an image is defined as:

$$
P(g)=\frac{N(g)}{M}
$$

where $M$ is the number of pixels in the image, and $N(g)$ is the number of pixels at grey level $g$. Take $L$ as the number of grey levels from 0 to 255 , the histogram features of the image including mean, standard deviation, skewness, kurtosis, energy, and entropy [24] based on the first-order histogram probability are formulated as expressed in Table 1. 
Table 1. The expression of histogram features of the image.

\begin{tabular}{ll}
\hline Index name & Expression \\
\hline Mean & $\overline{\mathrm{g}}=\sum_{g=0}^{L-1} g P(g)$ \\
Standard deviation & $\sigma_{g}=\sqrt{\sum_{g=0}^{L-1}(g-\bar{g})^{2} P(g)}$ \\
Skewness & $S=\frac{1}{\sigma_{g}^{3}} \sum_{g=0}^{L-1}(g-\bar{g})^{3} P(g)$ \\
Kurtosis & $K=\sum_{g=0}^{L-1} \frac{(g-\bar{g})^{3}}{\sigma^{4}}$ \\
Energy & ENERGY $=\sum_{g=0}^{L-1}[P(g)]^{2}$ \\
Entropy & ENTROPY $=-\sum_{g=0}^{L-1} P(g) \log _{2}[P(g)]$ \\
\hline
\end{tabular}

Generally, these features can be further selected and fused by feature fusion algorithms (e.g. principal component analysis) in order to decrease computational complexity and better discriminate different machine conditions. The selected and fused features are then fed into classifiers (e.g. support vector machine and Naïve Bayes classifier) for machinery defect diagnosis.

\section{EXPERIMENT STUDIES}

A series of experiments on a rotor test rig are performed to evaluate the effectiveness of the proposed infrared thermal image analysis method. Experimental analysis results are discussed below.

\subsection{Experimental Setup}

The experimental setup is shown in Fig. 3, which consists of a variable speed controller, a rotor test rig, an infrared thermal camera, and a GUI in the computer. The motor runs at 6,000 
RPM to drive the rotor system. An infrared thermal camera with model FLIR E50 is used to the capture the temperature distribution of the rotor test rig. Six different machinery conditions including normal state, mass unbalance, shaft misalignment, rotor radial rubbing, base looseness, and coupling unbalance and misalignment fault are simulated as listed in Table 2.

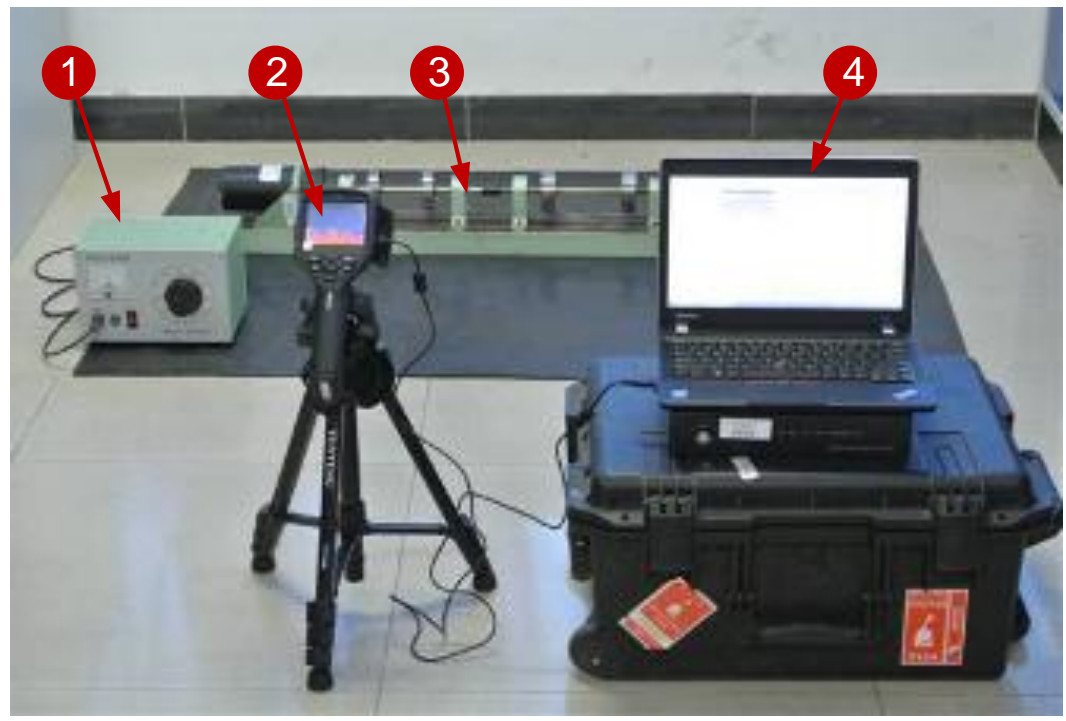

Fig. 3. Experimental setup of Rotor test rig, 1) variable speed controller, 2) infrared thermal camera, 3) rotor test rig, and 4) GUI in computer.

Table 2. Description of different conditions in rotor test rig.

\begin{tabular}{l|l}
\hline Condition description & Abbreviation \\
\hline Normal & NL \\
Mass unbalance & MU \\
Shaft misalignment & SM \\
Rotor radial rubbing & RR \\
Base looseness & BL \\
Coupling unbalance and misalignment faults & $\mathrm{CF}$ \\
\hline
\end{tabular}

In the rotor test rig, two steel shafts with $20 \mathrm{~mm}$ diameters are connected by a flexible couple which can be used to adjust the shaft misalignment. The rotating discs in the shaft are equipped with screwed holes, and extra mass can be installed to create the unbalance condition. The base looseness can be simulated by unfasten the bolts. A plastic sticker is used to simulate 
the rotor rubbing fault condition. The captured thermal image is shown in Fig. 4a. To improve the computational efficiency of presented method, the captured colour thermal images are transformed into grey-scale images for subsequent analysis. The grey image is shown in Fig. 4 with its scale in the range of $[0,255] .20$ images under each machinery condition are taken, and the examples of images under different machinery conditions are shown in Fig. 5.

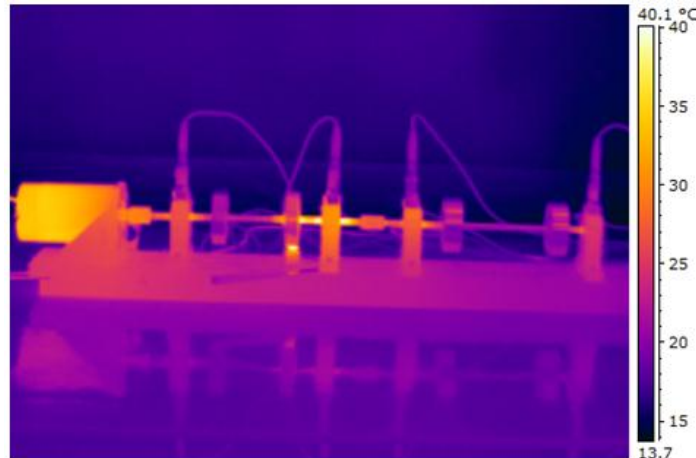

(a)

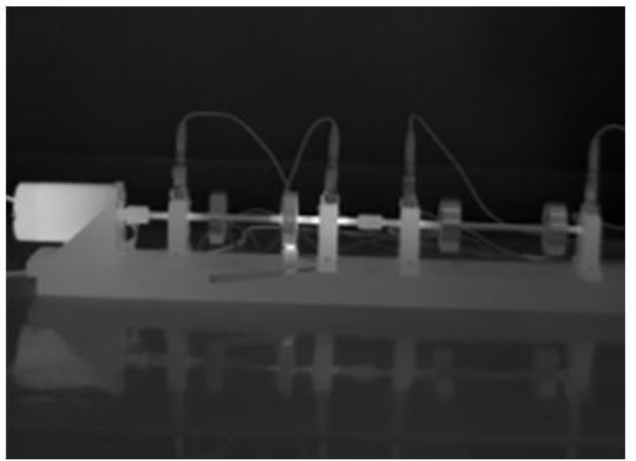

(b)

Fig. 4. Illustration of thermal image transformation, a) orignal thermal image, b) transformed grey image.

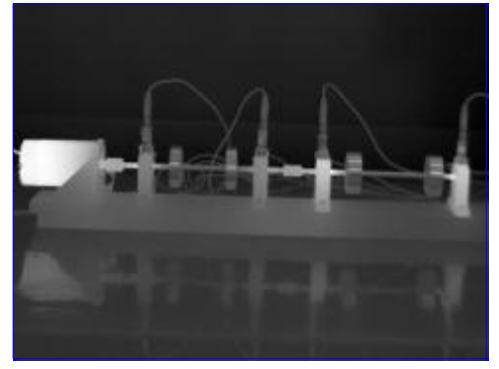

(a)

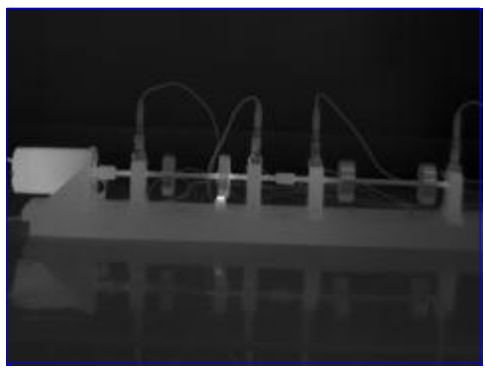

(d)

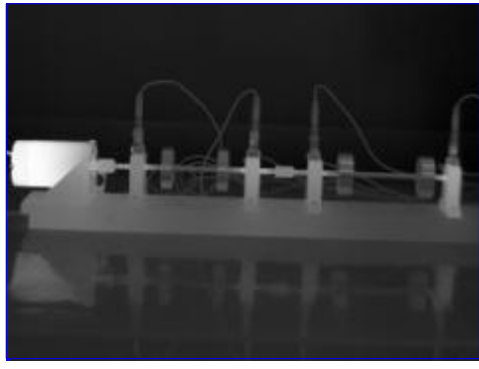

(b)

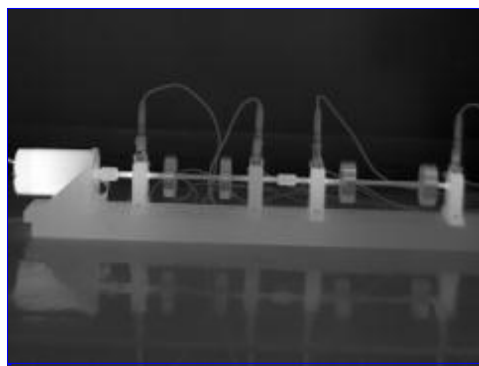

(e)

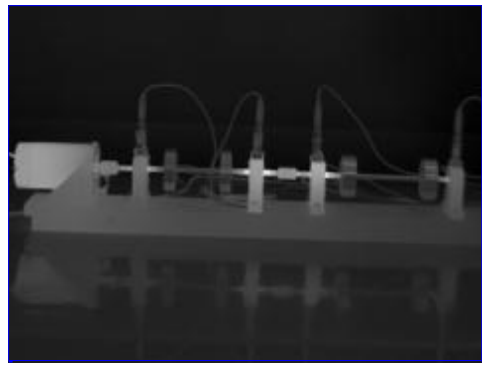

(c)

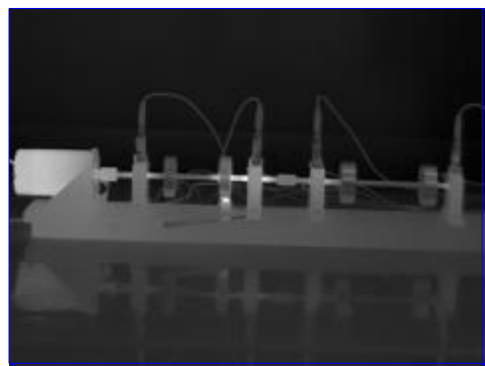

(f)

Fig. 5. Representative thermal images under different machinery conditions, a) normal, b) mass unbalance, c) shaft misalignment, d) rotor radial rubbing, e) base looseness, f) coupling unbalance and misalignment faults. 


\subsection{Effects of Segmentation Sizes}

The thermal image is segmented into different sizes, ranging from $1 \times 1$ to $18 \times 24$ blocks. Fig. 6 illustrates the examples of different segmented images such as $1 \times 1,3 \times 10,5 \times 15$, and 7 $\times 20$. One representative region for one machinery condition is selected according to the highest dispersion degree criterion as illustrated in Fig. 7 (e.g. region selection for $5 \times 15$ blocks). In the left of each sub-figure, it shows the dispersion degree calculated from the segmented image regions as shown in the right part of sub-figure. The segmented image region with highest dispersion degree will be selected as the image of interest.

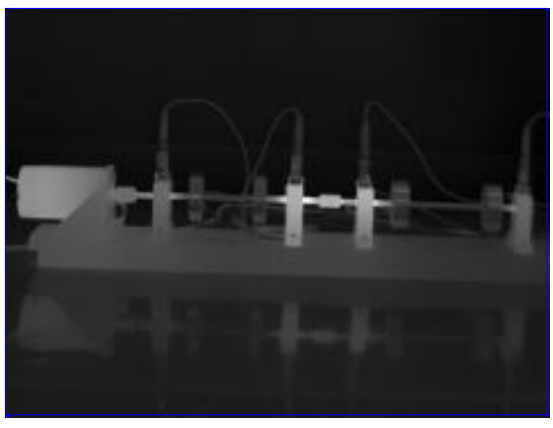

(a)

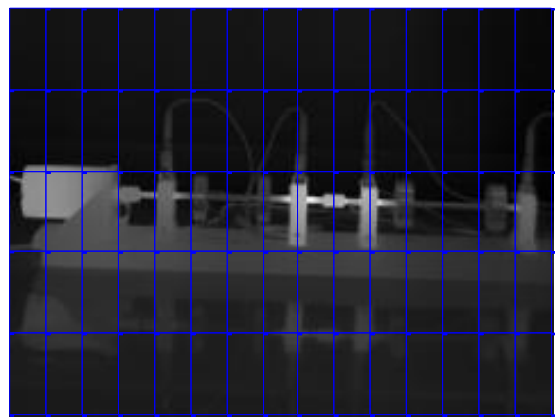

(c)

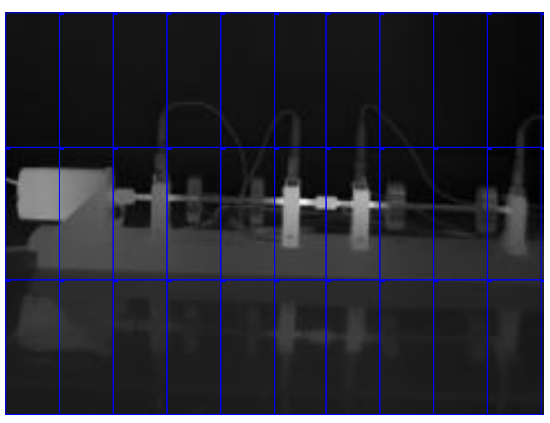

(b)

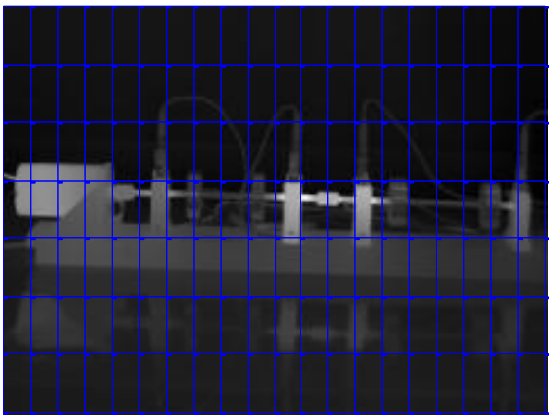

(d)

Fig. 6. Illustration of different image segmentations, a) $1 \times 1$, b) $3 \times 10$, c) $5 \times 15$, d) $7 \times 20$. 


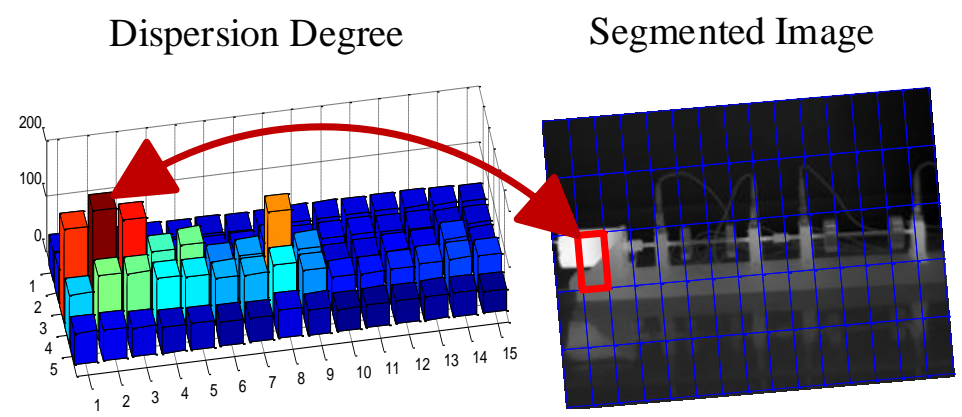

(a)

Dispersion Degree

Segmented Image

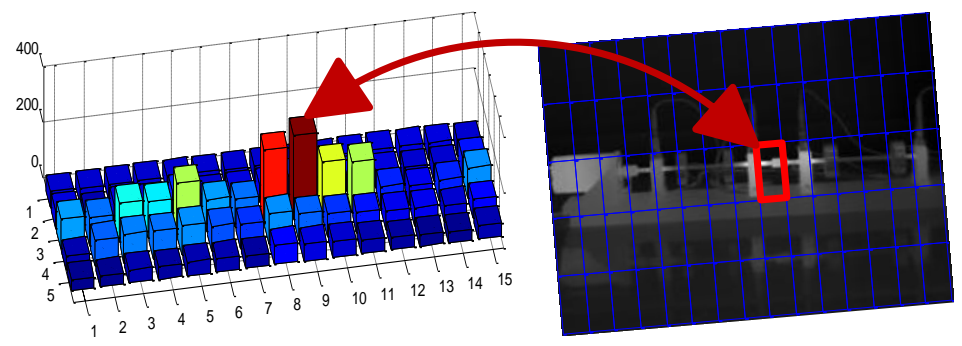

(b)

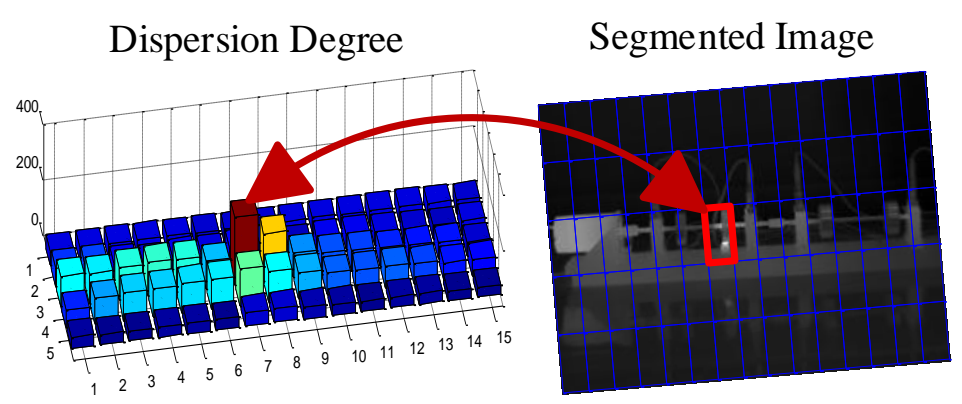

(c)

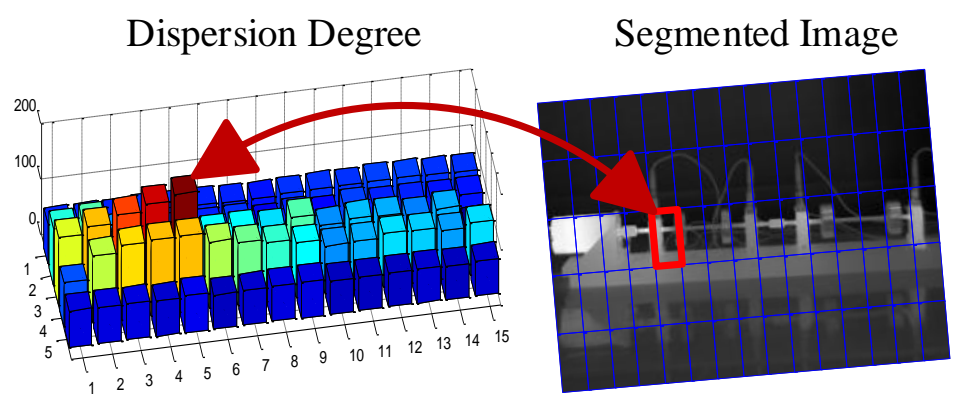

(d)

Fig. 7. Representative region for each independent fault based on dispersion degree criterion, a) mass unbalance, b) shaft misalignment, c) rotor radial rubbing, d) base looseness. 
Take the shaft misalignment fault as an example, the obtained color thermal image is shown in Fig. 8b. The thermal image acquired under machinery normal condition as shown in Fig. 8a is used for comparison. Since the shaft misalignment causes extra cyclic loads apply to the coupling, the temperatures of coupling and bearing supports nearby get increased as demonstrated in Fig. 8b. Next, the grey images are then transformed and segmented (e.g. $5 \times 15)$ as shown in Fig. 8d. According to Eq. 1, the dispersion degrees of different segmented regions are calculated as shown in Fig. 8c. Then, the region with the highest dispersion degree is selected as the region of interest.

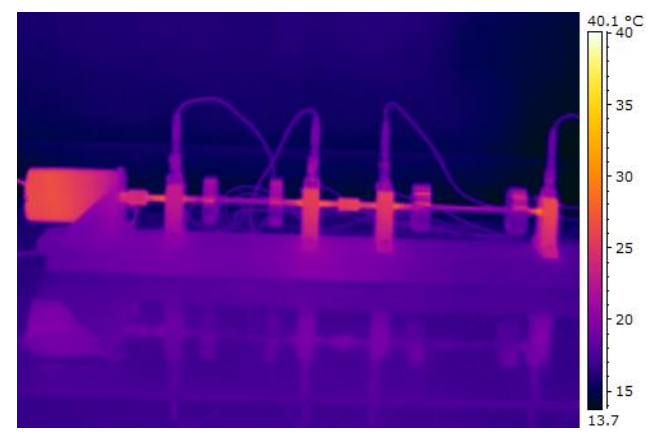

(a)

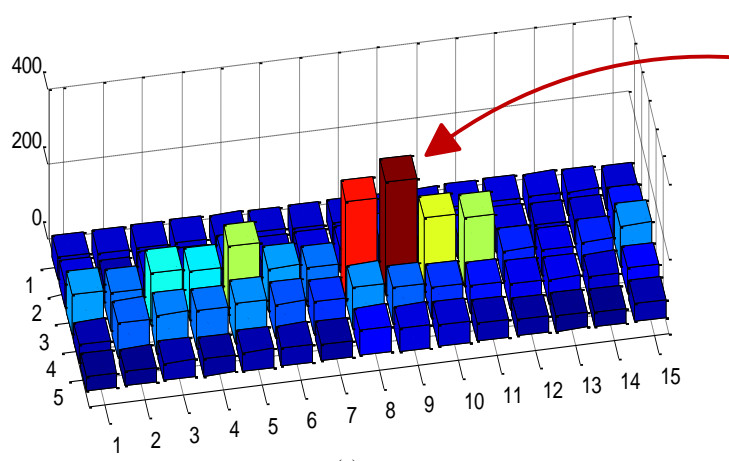

(c)

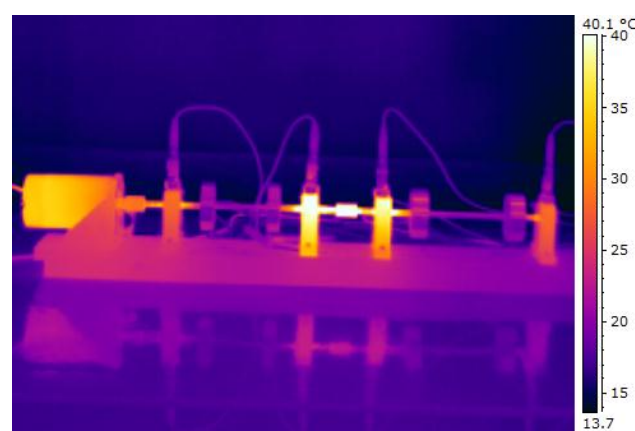

(b)

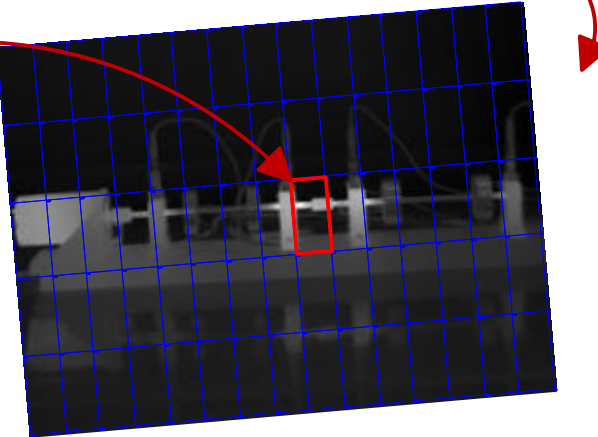

(d)

Fig. 8. Illustration of selection of segmented region according to highest dispersion degree under shaft misalignment condition.

Considering four independent faults in this experimental study, four representative regions which can cover the machinery conditions are selected and combined as the information of 
interest. The features as shown in Table 1 are calculated and extracted from the selected regions of thermal images. Feature reduction technique (e.g. principal component analysis) is then applied to the extracted features to select the relevant features and to eliminate the redundant features. The selected features with less dimensions are also beneficial to the computational efficiency of machine learning models. The selected feature number is determined by the accumulation contribution rate of principal components as shown in Fig. 9. It can be found that the accumulation contribution rate of first three principal components is higher than $90 \%$ and reflect most information of original feature space. Thus, the first three principal components are selected by projecting the six histogram features into three dimensions as shown in Fig. 10. The defect classification results tend to be clustered using PCA in a three-dimensional feature space under different types of segmentations. By comparing the cluster center and overlap, it can be found that the selected features are separated best under the segmentation size of $5 \times 15$.

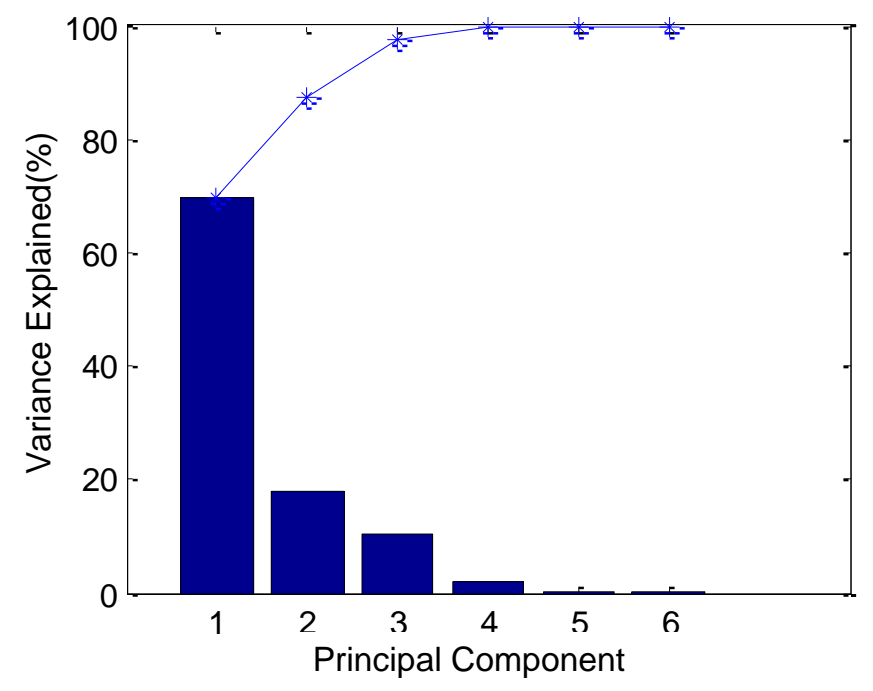

Fig. 9. The contribution rate of principal components. 

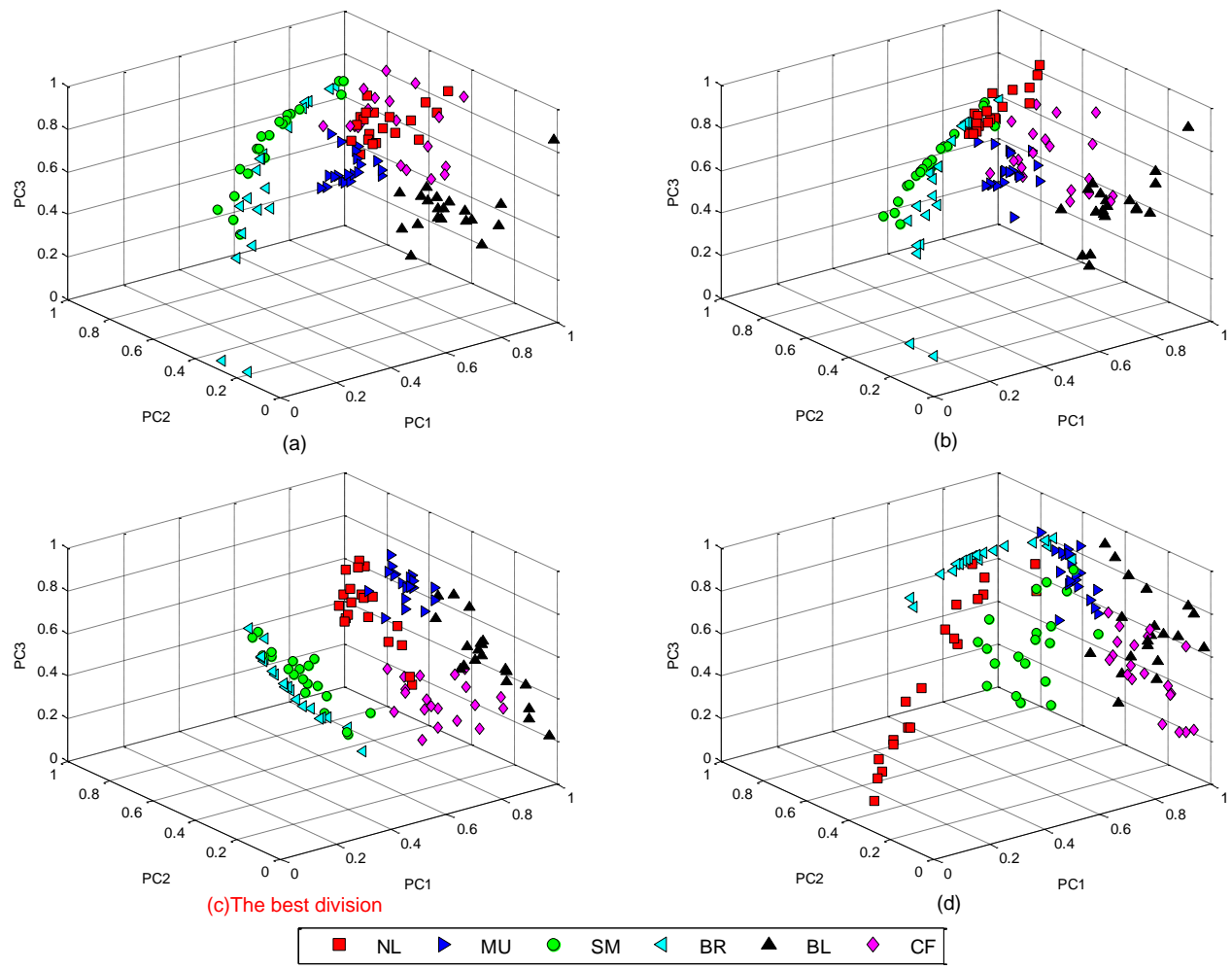

Fig. 10. Selected features under different divisions, a) $1 \times 1$, b) $3 \times 10$, c) $5 \times 15$, d) $7 \times 20$.

Next, the selected features are fed into Naïve Bayes classifier and support vector machine separately for machinery defect classification. The classification accuracies of Naïve Bayes and support vector machine under different segmentation conditions are shown in Fig. 11. As the segmentation number increases, the classification accuracy rises firstly, reaches the highest accuracy at the segmentation $5 \times 15$, and then decreases. With the presented segmented thermal image analysis, the classification accuracy of Naïve Bayes increases from $58 \%$ of the original image to $91.67 \%$ of the segmentation $5 \times 15$, while the classification accuracy of support vector machine increases from $42 \%$ of the original image to $90.67 \%$ of the segmentation $5 \times 15$. 


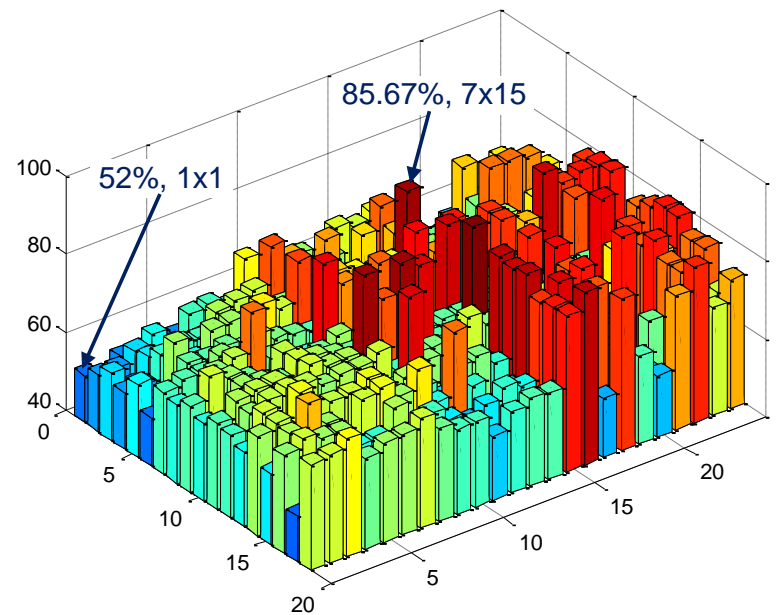

(a) Naïve bayes without PCA

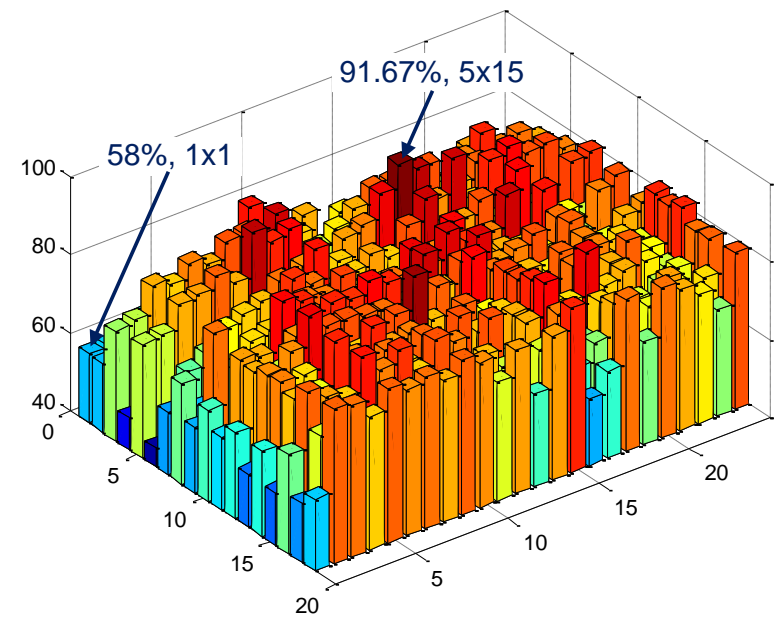

(c) Naïve bayes after PCA

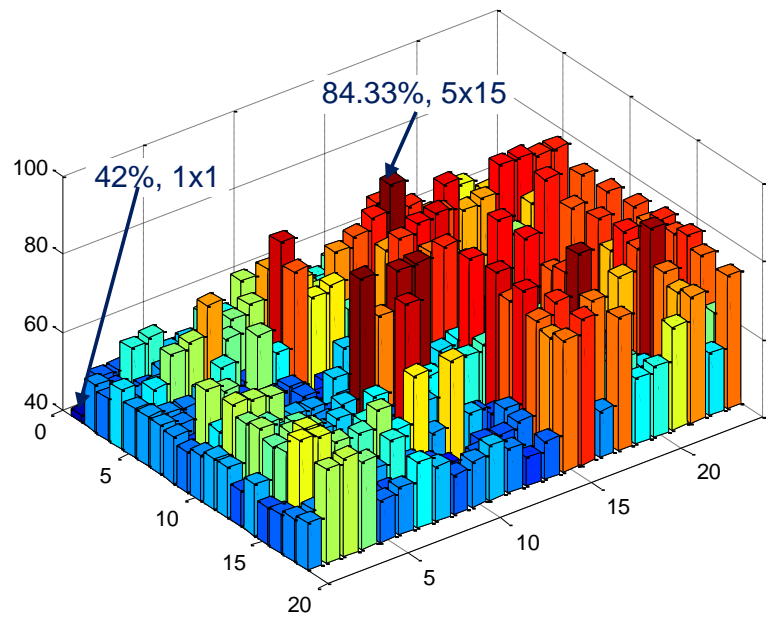

(b) Support Vector Machine without PCA

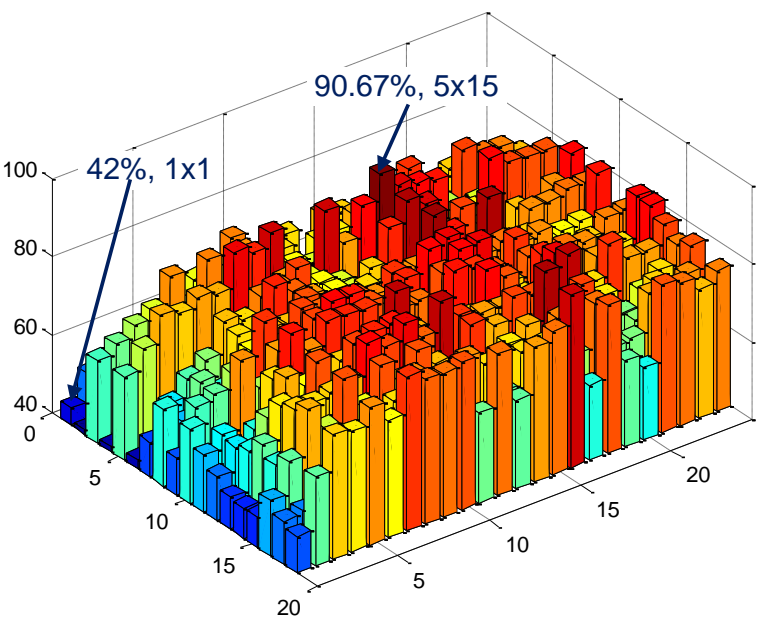

(d) Support Vector Machine after PCA

Fig. 11. The classification accuracy of Naïve Bayes and support vector machine under different segmentation sizes.

\section{DISCUSSIONS}

The defect classification is investigated under three different cases including using original image, segmented image analysis, and segmented image analysis with feature reduction. The classification results using Naïve Bayes and support vector machine are compared in Table 3.It can be found the presented segmented image analysis method with feature reduction yields best performance in terms of classification accuracy and computational time. 
Table 3. Comparison of Classification Results

\begin{tabular}{ccccccc}
\hline \multirow{2}{*}{ Classifier } & \multicolumn{2}{c}{ Original Image } & \multicolumn{2}{c}{ Segmented Method } & \multicolumn{2}{c}{ PCA-Segmented Method } \\
\cline { 2 - 7 } & Accuracy & Time & Accuracy & Time & Accuracy & Time \\
& $(100 \%)$ & $(\mathrm{s})$ & $(100 \%)$ & $(\mathrm{s})$ & $(100 \%)$ & $(\mathrm{s})$ \\
\hline Naïve Bayes & 58 & 0.0792 & 85.67 & 0.0727 & 91.67 & 0.0582 \\
Support Vector Machine & 42 & 0.9196 & 84.33 & 0.8695 & 90.67 & 0.7073 \\
\hline
\end{tabular}

From the above experimental analysis, it is found that the classification accuracy of machinery defect diagnosis increases using the segmented infrared thermal image analysis. It reaches highest accuracy under the segmentation $5 \times 15$. It is worthy to interpret the selected region from the machinery physical properties. As shown in Fig. 7, the excessive load is applied on the motor to cause the increase of motor temperature under the mass unbalance condition, thus the region at the motor location is selected as the representative region for mass unbalance based on the dispersion degree criterion. Similarly, with the shaft misalignment, the vibration of the shaft couple causes the temperature increase at the location of shaft couple. The temperature of the rotating disc increases due to the rotor radial rubbing defect. The base looseness causes excessive vibration of coupling between the motor and the shaft, resulting in increased temperature of the region at this location, which is selected as representative region for base looseness. Therefore, it is reasonable to take the segmented thermal image analysis method by emphasizing fault-related regions and discarding irrelevant background information.

In infrared image based fault diagnosis, the main influence factors involve the temperature variation and environment, besides the low contrast and resolution in the infrared image itself. The presented method can remove irrelevant background information and emphasize fault related regions, thus it is less affected by the environment. Meanwhile, the temperature variation of the environment affects little because the mass and energy of the equipment are great, and the temperature difference between the equipment and the environment are much larger. It can be 
concluded that the proposed method is applicable in different scenarios. To further evaluate the robustness of proposed method, more tests would be done in the future studies.

\section{CONCLUSIONS}

In this study, a new feature enhancement method mainly composed by image segmentation and region selection in infrared image analysis is proposed for machinery fault diagnosis. Experimental studies on a rotor test bed have been performed to demonstrate the effectiveness of the presented method. The conclusions can be drawn as follows.

(1) A dispersion degree criterion is formulated to guide the fault-related region selection in infrared thermal image analysis.

(2) The rationality of region selection criterion is discussed through the physical interpretation of machinery failure modes by correlating selected regions with machinery failure modes.

(3) The presented segmented infrared thermal image analysis method can remove irrelevant background information and emphasize fault related regions. Thus the accuracy of machinery defect diagnosis is improved.

\section{ACKNOWLEDGEMENTS}

This research acknowledges the financial support provided by National Science foundation of China (No.51005247), and Science Foundation of China University of Petroleum, Beijing (No. 2462014YJRC039 and 2462015YQ0403). Valuable comments from anonymous reviewers are greatly appreciated to improve the paper's quality. 


\section{APPENDIX A}

The Naive Bayesian Classifier is commonly used for classification problems in machine learning and data mining fields due to its simplicity and impressive classification accuracy. It is a simple probabilistic approach to achieve classification based on Bayes' theorem. Given an

unclassified object with its feature vector $x$, Naïve Bayes classifier recognizes this object $x$ as the class $y_{\mathrm{i}}$ which has the highest posterior probability $P\left(y_{i} \mid x\right)$ conditioned on $x$ [25]. According to Bayes theorem, it can be expressed as

$$
P\left(y_{i} \mid x\right)=\frac{P\left(x \mid y_{i}\right) P\left(y_{i}\right)}{P(x)}
$$

Since $P(x)$ is the same for all classes, and $P\left(y_{i}\right)$ can be determined from the training data set, the crucial part is to determine the conditional probability $P\left(x \mid y_{i}\right)$. The determination of $P\left(x \mid y_{i}\right)$ is usually computational expensive and requires large training set, the naïve Bayes model simplifies the estimation of $P\left(x \mid y_{i}\right)$ by assuming:

$$
P\left(x \mid y_{i}\right)=\prod_{k=1}^{N} P\left(x_{k} \mid y_{i}\right)
$$

The Naïve Bayes classifier combines this model with decision rule by choosing the maximum posteriori probability. The corresponding classifier is the function classify defined as follows.

$$
f=\arg \max P\left(y_{i}\right) \prod_{i=1}^{N} P\left(x_{i} \mid y_{i}\right)
$$

\section{APPENDIX B}

As a powerful machine learning method for classification of small samples and high dimensions, support vector machine (SVM) is initially presented by Vapnik in the last decade of the 20th century based on statistical learning theory and structural risk minimization principle [26]. SVM transforms the original feature space into a higher dimensional space to determine an 
optimal hyperplane by maximizing the separation distances among the classes. Given an input training data set $\vec{x} \in \chi$, the transformed higher dimensional feature space can be obtained as:

$$
\vec{x}^{\prime}=\phi(\vec{x})
$$

where $\phi$ is the transformation function. Assuming two classes $y \in\{1,-1\}$ labelled as positive class $y_{i}=1$ and negative class $y_{i}=-1$, a hyperplane $f\left(x^{\prime}\right)=0$ can be determined as:

$$
f\left(\vec{x}^{\prime}\right)=\vec{w}^{T} \bar{X}^{\prime}+b=\sum_{j=1}^{n} w_{j} x_{j}^{\prime}+b=0
$$

where $\vec{w}$ is a n-dimensional vector and $b$ is a scalar. The vector $\vec{w}$ and the scalar $b$ are used to define the position of the separating hyperplane.

This hyperplane is built to maximize the distance among the closest classes through the following optimization.

$$
\max _{w \in R, b \in R} D, \text { subject to } y_{i}\left(\vec{w}^{T} x_{i}^{\prime}+b\right) \geq D, \forall i
$$

where $\mathrm{D}$ is the distance of the closest class to the hyperplane and can be set as $1 /\|\vec{w}\|$ after normalization. Taking into account the noise with slack variables $\xi_{i}$ and the error penalty C, Eq. (B-2) can be rewritten as:

$$
\min _{w, \xi \in R^{n}, b \in R}\left\{\frac{1}{2}\|\vec{w}\|^{2}+C \sum_{i=1}^{N} \xi_{i}\right\}, \text { subject to } \xi_{i} \geq 0, y_{i}\left(\vec{w}^{T} \phi\left(x_{i}\right)+b\right) \geq 1-\xi_{i}, \forall i
$$

Then the hyperplane can be determined as the following sign function $(\operatorname{sgn}(t)=1$ for $t \geq 0$, and $\operatorname{sgn}(t)=-1$ for $t<0)$.

$$
f(\vec{x})=\operatorname{sgn}\left(\sum_{i, j=1}^{N} y_{i} \alpha_{i} \phi\left(x_{i}\right)^{T} \phi\left(x_{j}\right)+b\right)
$$


Table B-1. Formulation of kernel functions.

\begin{tabular}{ll}
\hline Kernel & Expression \\
\hline Linear & $\vec{x}^{T} \cdot x_{j}$ \\
Polynomial & $\left(\gamma \vec{x}^{T} \cdot x_{j}+C\right)^{d}, \gamma>0$ \\
Gaussian RBF & $\exp \left[-\frac{\left\|\vec{x}-x_{j}\right\|^{2}}{2 \gamma^{2}}\right]$ \\
Sigmoid & $\tanh \left[\gamma \vec{x}^{T} x_{j}+C\right]$ \\
\hline
\end{tabular}

Then, the hyperplane function can be determined by kernel function $K\left(x_{i}, x_{j}\right)=\phi\left(x_{i}\right)^{T} \phi\left(x_{j}\right)$ by computing the inner products without specifying the explicit form of the transformation function. Different kernels can be formulated as listed in Table B-1, where $\gamma$ is a kernel parameter, $\mathrm{C}$ is a cost parameter in the kernel function, and $d$ denotes the degree of the polynomial function. Specifically, the Gaussian RBF kernel is used in this study due to its popularity and good performance in machinery condition monitoring. Accordingly, the associated decision function is expressed as [27]:

$$
f(\vec{x})=\operatorname{sgn}\left(\sum_{i, j=1}^{N} y_{i} \alpha_{i} K\left(x_{i}, x_{j}\right)+b\right)
$$

\section{REFERENCES}

[1] R. Teti, K. Jemielniak, G O'Donnel, D. Dornfeld, Advanced monitoring of machining operations, CIRP Annals-Manufacturing Technology, 59(2010)717-739.

[2] A.K. Jardine, D. Lin, and D. Banjevic, A review on machinery diagnostics and prognostics implementing condition-based maintenance, Mechanical systems and signal processing, 20(7)(2006)1483-1510.

[3] J. Wang, R.X. Gao, and R. Yan, Multi-scale enveloping order spectrogram for rotating 
machine health diagnosis, Mechanical Systems and Signal Processing, 46(1)(2014)28-44.

[4] Y. Lei, J. Lin, Z. He, M. Zuo, A review on empirical mode decomposition in fault diagnosis of rotating machinery, Mechanical Systems and Signal Processing, 35(1-2)(2013)108-126.

[5] S. Osman, W. Wang, An enhanced Hilbert-Huang Transform technique for bearing condition monitoring, Measurement Science and Technology, 24(2013)1-14.

[6] Z.N. Li, J. Tang, Q.S. Li, Optimal sensor locations for structural vibration measurement, Applied Acoustics, 65(8)(2004)807-818.

[7] A. Prabhakaran, C.R. Jagga, Condition monitoring of steam turbine-generator through contamination analysis of used lubricating oil, Tribology International, 32(3)(1999)145-152.

[8] S. Bagavathiappan, B.B. Lahiri, T. Saravanan, J. Philip, T. Jayakumar, Infrared thermography for condition monitoring - a review, Infrared Physics \& Technology, 60(2013)35-55.

[9] A.M. Younus, B.S. Yang, Intelligent fault diagnosis of rotating machinery using infrared thermal image, Expert Systems with Applications, 39(2012)2082-2091.

[10] M.S. Jadin, S. Taib, Recent progress in diagnosing the reliability of electrical equipment by using infrared thermography, Infrared Physics \& Technology, 55(2012)236-245.

[11] M.J. Picazo-Rodenas, R. Royo, J. Antonino-Daviu, J. Roger-Folch, Use of the infrared data for heating curve computation in induction motors: application to fault diagnosis, Engineering Failure Analysis, 35(2013)178-192.

[12] A. Kylili, P.A. Fokaides, P. Christou, S.A. Kalogirou, Infrared thermography (IRT) applications for building diagnostics: a review, Applied Energy, 134(2014)531-549.

[13] P. Broberg, Surface crack detection in welds using thermography, NDT\&E International, 57(2013)69-73.

[14] C. Meola, A new approach for estimation of defects detection with infrared thermography, Materials Letters, 61(2007)747-750.

[15] B.B. Lahiri, S. Bagavathiappan, T. Jayakumar, J. Philip, Medical applications of infrared thermography: a review, Infrared Physics \& Technology, 55(2012)221-235.

[16] U.R. Acharya, J.H. Tan, J. E.W. Koh, V.K. Sudarshan, S. Yeo, C.L. Too, C.K. Chua, E.Y.K. $\mathrm{Ng}$, L. Tong, Automated diagnosis of dry eye using infrared thermography images, Infrared Physics \& Technology, 71(2015)263-271.

[17] B.F. Jones, A reappraisal of the use of infrared thermal image analysis in medicine, IEEE Transactions on Medical Imaging, 17(6)(1998)1019-1027.

[18] B.B. Lahiri, S. Bagavathiappan, C. Soumya, V. Mahendran, V.P.M. Pillai, John Philip, T. Jayakumar, Infrared thermography based defect detection in ferromagnetic specimens using a low frequency alternating magnetic field, Infrared Physics \& Technology, 64 (2014)125-133.

[19] C. Meola, G.M. Carlomagno, Recent advances in the use of infrared thermography, Measurement Science \& Technology, 15(9)(2004)27-58.

[20] B.B. Lahiri, S. Bagavathiappan, R. Shunmugasundaram, John Philip, T. Jayakumar, Measurement of annular air-gap using active infrared thermography, Infrared Physics \& Technology, (61)(2013)192-199.

[21] A. Taheri-Garavand, H. Ahmadi, M. Omid, S.S. Mohtasebi, K. Mollazade, A.J.R. Smith, G.M. Carlomagno, An intelligent approach for cooling radiator fault diagnosis based on infrared thermal image processing technique, Applied Thermal Engineering, 87(2015)434-443. 
[22] G.M. Lim, D.M. Bae, and J.H. Kim, Fault diagnosis of rotating machine by thermography method on support vector machine, Journal of Mechanical Science and Technology, 28(8)(2014)2947-2952.

[23] V.T. Tran, B.S. Yang, F. Gu, A. Ball, Thermal image enhancement using bi-dimensional empirical mode decomposition in combination with relevance vector machine for rotating machinery fault diagnosis, Mechanical Systems and Signal Processing, 38(2013)601-614.

[24] H.N. Jiang, L. Zeng, B. Bi, A comprehensive method of contour extraction for industrial computed tomography images, optics and Lasers in Engineering, 51(3)(2013)286-293.

[25] R.R. Yager, An extension of the naïve Bayesian classifier, Information Sciences 176(2004):577-588.

[26] V.N. Vapnik, 1999, The nature of statistical learning theory, New York: Springer.

[27] J. Wang, S. Liu, R. Gao, R. Yan, Current envelope analysis for defect identification and diagnosis in induction motors, Journal of Manufacturing Systems, 31(4)(2012)380-387. 